\title{
Complementary Alliances with Endogenous Fleets and Load Factors
}

\author{
Achim l. Czernya \\ Vincent A.C. van den Bergb \\ Erik T. Verhoefo
}

a Hong Kong Polytechnic University, Hong Kong, PR China;

${ }^{b}$ Faculty of Economics and Business Administration, VU University Amsterdam, and Tinbergen Institute, the Netherlands. 
Tinbergen Institute is the graduate school and research institute in economics of Erasmus University Rotterdam, the University of Amsterdam and VU University Amsterdam.

More TI discussion papers can be downloaded at http://www.tinbergen.nl

Tinbergen Institute has two locations:

Tinbergen Institute Amsterdam

Gustav Mahlerplein 117

1082 MS Amsterdam

The Netherlands

Tel.: +31(0)20525 1600

Tinbergen Institute Rotterdam

Burg. Oudlaan 50

3062 PA Rotterdam

The Netherlands

Tel.: +31(0)10 4088900

Fax: +31(0)10 4089031 


\title{
Complementary Alliances With Endogenous Fleets and Load Factors ${ }^{1}$
}

\author{
Achim I. Czerny ${ }^{2}$, Vincent A. C. van den Berg ${ }^{3}$ and Erik T. Verhoef ${ }^{4}$
}

January 3, 2016

\footnotetext{
${ }^{1}$ We thank Serguei Netessine, Sarah Wan, Anming Zhang, the participants of the EUREKA seminar at the VU University Amsterdam for helpful comments and suggestions. Partial financial support from the European Research Council (ERC, AdG Grant $\sharp 246969$ OPTION) is gratefully acknowledged.

${ }^{2}$ Department of Logistics and Maritime Studies, Hong Kong Polytechnic University, Hong Kong, achim.czerny@polyu.edu.hk.

${ }^{3}$ Department of Spatial Economics, VU University Amsterdam, and Tinbergen Institute, The Netherlands, v.a.c.vanden.berg@vu.nl

${ }^{4}$ Department of Spatial Economics, VU University Amsterdam, and Tinbergen Institute, The Netherlands, e.t.verhoef@vu.nl.
} 


\begin{abstract}
This paper analyzes the effect of carrier collaboration on fleet capacity, fleet structures in terms of the number and the size of vehicles, and load factors. The model features complementary networks, scheduling, price elastic demands, and demand uncertainty. For the case of a given number of vehicles, the analysis shows that carrier collaboration increases vehicle sizes (thus, fleet capacity) if marginal seat costs are low while fleet capacity remains unchanged if marginal seat costs are high. If both vehicle sizes and vehicle numbers can be varied, then collaboration will always increase vehicle numbers and fleet capacity, while the effects on vehicle sizes and, thus, also load factors, are ambiguous and therewith hard to predict. Numerical simulations indicate that collaboration increases expected load factors also when the number of vehicles is endogenous.
\end{abstract}

Keywords: Alliances; fleet capacity; load factors; scheduling; uncertainty. 


\section{Introduction}

A single trip typically involves various carriers with complementary networks. Consider an air trip as an example. Passengers may use the taxi in order to travel from their home to the airport, and fly from there with one airline to a hub airport where they transfer to the flight of a different airline to a third airport and there travel by rail or metro to their final destination. Key questions for the involved carriers are how to choose fares, service qualities and fleets in such an environment. Because carriers are often exempted from antitrust law (for example, this is true for airlines and liner shipping companies), another question is how to evaluate carrier collaboration from the social perspective. This perspective is relevant for policy makers but also for firms that would like to receive exemption from antitrust law in the future.

There is striking theoretical and empirical evidence that carrier collaboration can lower fares and at the same time increase profits of the involved carriers (e.g., Brueckner and Whalen, 2001; Brueckner, 2001; Bamberger et al., 2004; Armantier and Richards, 2008). Collaboration can eliminate double marginalization and, thus, reduce fares for passengers who are served by multiple carriers (called interline passengers). ${ }^{1}$ An important side effect is that the reduction in fares can increase social welfare defined by the sum of profits and consumer surplus. This provides one explanation why carriers may be exempted from antitrust law. Zhang and Zhang (2006) show that rivalry between complementary alliances can further enhance social welfare, because of a strategic effect that results in a higher degree of alliance, and greater output levels, than would be the case in the absence of such rivalry. In their study, the degree of alliance is measured by the share of the partner airlines' profits that airlines take into account when they decide upon fares. These shares may have the interpretation of "prorates" considered in further detail by $\mathrm{Hu}$ et al. (2013), who analyzed equilibrium shares of airline fares (prorates) for given airline fares and stochastic demand. Let schedule delays describe the difference between the passengers' most preferred and actual travel times (e.g., Miller, 1972; Douglas and Miller, 1974). Adler and Hanany (2015) concentrate on parallel airline networks and capture the effect of collaboration on schedule delays in their analysis. They find that collaboration can increase consumer and social surplus through an increase in airline frequency supply.

\footnotetext{
${ }^{1}$ Czerny (2009) shows that non-stop passengers may be charged a higher fare with carrier collaboration because with carrier collaboration carriers can price discriminate between non-stop and interline passengers (also see Zhang and Czerny (2012) for a discussion of this issue).
} 
A common feature of this literature strand is that either capacities or load factors, i.e., the ratio of passengers over seat capacity, is considered as fixed.

The effect of carrier collaboration on load factors is of interest for several reasons. If carrier collaboration does not only lower fares but also increases load factors, this may bring another profit and welfare gain as they allow carriers to more efficiently use their fleet. Load factors are also commonly used as measures for the market performance of carriers. Consistent financial information to evaluate carrier performance is sometimes unavailable. This is particularly true for international markets where different accounting standards may apply, and in such situations researchers have reverted back to non-financial information such as load factors to (indirectly) analyze the effects of carrier collaboration on profits (Lazzarini, 2007). ${ }^{2}$ Two conditions must be satisfied for non-financial information being a good substitute for financial information: Carriers and policy makers need to understand the relationships between (i) carrier collaboration and load factors, and between (ii) load factors, profits and social welfare.

The evidence for the relationship between carrier collaboration and load factors, point (i), is mixed. ${ }^{3}$ Chen and Chen (2003) abstract away from schedule effects and use a newsboy model with exogenous fares and uncertain but price insensitive demand to derive hypotheses about the effect of carrier collaboration on load factors. They find theoretical and empirical evidence which indicates that load factors are unchanged by carrier collaboration in the case of complementary networks and increasing in carrier collaboration in the case of parallel networks. Iatrou and Alamdari (2005) provide survey data, where carriers state that collaboration increases load factors. Lazzarini (2007) empirically studies the impact of airline alliances on carriers' operational performance measured by load factors. He finds that both alliance membership or close relations to other airlines that are members of an alliance can increase load factors. Li and Netessine (2011), consider airline collaborations for parallel networks, and empirically find that load factors are reduced by carrier collaboration. With the exception of Chen and Chen (2003), all the literature on load factors mentioned above is empirical. Again, this is partly because it is common in the theoretical literature to assume that fleet sizes are given or that vehicles are fully

\footnotetext{
${ }^{2}$ The difficulties to calculate load factors for airlines using available airline statistics is considered by Devrient et al. (2009).

${ }^{3}$ Viton (1986) is interested in the effect of deregulation on the industry from the policy viewpoint. In line with Douglas and Miller (1974), he considers load factors as a measure for service quality, and finds that load factors remained too low after a year of deregulation.
} 
booked. ${ }^{4}$ The latter essentially means that outputs and capacities are the same (e.g., Hu, 2010).

The evidence for the relationship between load factors and carrier profits, point (ii), is also mixed. If vehicle sizes are fixed, load factors may implicitly, through frequency, determine service quality in terms of schedule delays and, thus, demand and revenue potentials. This may suggest that there is a negative relationship between profits and load factors. On the other hand, if vehicle sizes are endogenous, load factors (together with vehicle sizes and fares) determine average cost per passenger and thus the degree to which carriers may be able to exploit scale economies. This may suggest that there is a positive relationship between profits and load factors. ${ }^{5}$

Altogether, this indicates that a better understanding of the role of load factors and fleets for the evaluation of carrier collaboration is required. The main contribution of the present study is therefore to analyze the effect of carrier collaboration on the fleet size, the composition of fleets in terms of the number and the size of vehicles, and load factors when carrier networks are complementary.

\section{The Basic Model}

There are two risk neutral and symmetric carriers that operate perfectly complementary networks. Think of two equidistant city pairs A-B and B-C, where the connection between $\mathrm{A}$ and $\mathrm{B}$ is operated by carrier 1 and the connection between $\mathrm{B}$ and $\mathrm{C}$ is operated by carrier 2. The non-negative quantity of passengers traveling between $\mathrm{A}$ and $\mathrm{C}$ is denoted as $q$; i.e., there is no origin-destination travel on the short distances between A and B and between $\mathrm{B}$ and $\mathrm{C}$. Furthermore, there is no direct connection between $\mathrm{A}$ and $\mathrm{C}$ available, so that passengers have to use the services of both carriers 1 and 2 in order to reach their final destination.

Passenger benefits are denoted as $B$. To capture the notion of uncertainty it is assumed

\footnotetext{
${ }^{4}$ Brueckner and Zhang (2010) and Czerny (forthcoming) analyze the effect of emission charges on aircraft design when carriers choose the aircraft fleet in terms of the size and the number of aircraft so that load factors are endogenous. However, they abstract away from carrier collaboration and complementary networks (among other differences in the modeling).

${ }^{5}$ Behn and Riley (1999) empirically found a positive relationship between load factors and operating income and load factors and operating revenue. Graham et al. (1983) and Bailey et al. (1985) empirically demonstrate that when travel distance or market density increases, the airlines will operate larger aircraft at higher load factors.
} 
that benefits are concave, stochastic and given by $B=B(q ; \varepsilon)$ with $B^{\prime \prime}<0$ and $\partial B^{\prime} / \partial \varepsilon>0$ for $q>0$, i.e., inverse demand shifts up with $\varepsilon$ for all $q$ (primes denote partial derivatives). The term $\varepsilon$ is a random term that follows a Bernoulli distribution with zero expectation and standard deviation $\sigma>0$, which means that $\varepsilon=-\sigma, \sigma$, and that high and low demands will occur with the same probability. Fares are denoted as $p_{1}$ and $p_{2}$ for carriers 1 and 2, respectively, where the sum of these fares yields the total fare of traveling, denoted $\eta$ with $\eta=p_{1}+p_{2}$. Demand is determined by the equilibrium condition $B^{\prime}=\eta$, which implicitly defines the downward sloping demand function $D(\eta ; \varepsilon)$ with $D^{\prime}=1 / B^{\prime \prime}<0$ and $\partial D / \partial \varepsilon>0$.

The individual fleet sizes (capacities) are denoted $k_{1}$ and $k_{2}$ for carriers 1 and 2 , respectively. For example, capacity limits may be determined by the sizes of a given number of vehicles (if the vehicle numbers are normalized to 1, vehicle sizes are given by $\left.k_{i}\right)$. Airports where the quantity of flights are constrained by slots, which entitle airlines to make use of airport capacity at a specific point in time, justifies the scenario with fixed vehicle numbers (Czerny, Verhoef and Zhang, 2015). The existence of capacity limits together with the perfect complementarity of carrier networks means that the passenger quantity, $q$, is limited by the minimum capacity $Q$ with $Q=\min \left\{k_{1}, k_{2}\right\}$. Thus, $q \leq Q$, which is called the capacity constraint.

Operating costs are normalized to zero and independent of the demand-capacity ratio; i.e., there is no congestion. It may be increasingly difficult to increase an already large capacity because it may be increasingly difficult to find qualified staff or to rent additional infrastructure space required for the operation of larger vehicles. Letting $g$ with $g=g\left(k_{i}\right)$ denote the strictly convex capacity costs, it is therefore assumed that $g^{\prime}, g^{\prime \prime}>0$. The carrier profits denoted $\pi_{i}$ can be written as $\pi_{i}=p_{i} \cdot \min \{D(\eta ; \varepsilon), Q\}-g\left(k_{i}\right)$. The righthand side shows the revenue, which is constrained by capacity, less the capacity costs.

The time structure involves two stages: In the first stage, the carriers choose capacities $k_{1}$ and $k_{2}$, while the carriers choose fares $p_{1}$ and $p_{2}$ knowing the demand state, i.e., the value of the random error $\varepsilon$, in the second-stage. This sequential structure captures that pricing decisions can be easily changed relative to capacity decisions, which are typically more long term (Czerny, Verhoef and Zhang, 2015). This feature (among other features) distinguishes the present study from other studies, for example, $\mathrm{Hu}$, Caldenty and Vulcano (2013), who analyzed proration rates for given airline fares.

We distinguish between two scenarios called the independent carriers scenario and the 
alliance carriers scenario. In the independent carriers scenario, carriers choose fares and capacity to maximize own expected profit. In the alliance carriers scenario, fares and capacities are chosen in order to maximize joint expected profits. The two scenarios are solved backwards.

\section{$3 \quad$ Vehicle Sizes Endogenous (Vehicle Numbers Fixed)}

It is useful to distinguish between cases with "low" and "high" demand uncertainty, as measured by the standard deviation parameter $\sigma$. In the case of low demand uncertainty, stochastic demand fluctuations are relatively small so that the capacity constraint can be binding for both positive and negative values of $\varepsilon$ (i.e., for $\varepsilon=-\sigma, \sigma$ ). The case with relatively small demand fluctuations therefore implies expected load factors of one-hundred percent. ${ }^{6}$ To capture that expected load factors can be below one-hundred percent (which is a crucial element of the present analysis), sufficiently high demand uncertainty has to be considered and is the maintained assumption in the present study. More specifically, we will assume that the stochastic demand fluctuations determined by $\sigma$ are sufficiently high to ensure that the capacity constraint is binding only in the high state of demand (i.e., for $\varepsilon=\sigma$ ), thus, not binding in the low state of demand (i.e., for $\varepsilon=-\sigma$ ). We further assume that this is true with and without cooperation. The profits, denoted $\pi_{i}$, in the low state of demand can then be written as $\pi_{i}=p_{i} D(\eta ;-\sigma)-g\left(k_{i}\right)$. Derivation of profits in the high state of demand is more complicated because of the possibility of corner solutions, which will be discussed in detail below.

Independent carriers choose fares to maximize their own revenue. In the low state of demand, fares are then implicitly determined by the first-order conditions $\partial \pi_{i} / \partial p_{i}=$ $D(\eta ;-\sigma)+p_{i} D^{\prime}(\eta ;-\sigma)=0 .{ }^{7}$ But, this means that independent carriers ignore the effect of an increase in their fares on the other carrier's profit, where this effect is negative and determined by $p_{j} D^{\prime}(\eta ;-\sigma)<0$ with $j \neq i$, which reflects double marginalization. With carrier collaboration and joint profit maximization double marginalization can be eliminated, which leads to a reduction in the total fare of traveling $\eta$ relative to when

\footnotetext{
${ }^{6} \mathrm{Hu}(2010)$ concentrates on scenarios with relatively low demand uncertainty in the sense that aircraft are are always fully booked in his analysis of endogenous airline network structures.

${ }^{7}$ The second-order conditions are satisfied if the benefit function is sufficiently concave in the sense that that the elasticity of the slope of the inverse demand $B^{\prime}$ is strictly less than the absolute value of the demand slope $D^{\prime}=1 / B^{\prime \prime}$ (Czerny, Verhoef and Zhang, 2015).
} 
carriers are independent. That collaboration can lead to reduced prices relative to the prices charged by independent firms and products has first been shown by Spengler (1950) for the case of a vertical structure with upstream and downstream firms. The case of horizontal integration has been considered by, for example, Economides and Salop (1992), Brueckner and Whalen (2000), and Brueckner (2001).

To derive equilibrium fares for the high state of demand, it is useful to remember that in this demand state the capacity constraint is binding. If the capacity constraint would not be binding in the high-demand state, then carriers would clearly have invested into excess capacity because it is also not binding in the low state of demand by assumption. This however can not be optimal given that marginal capacity costs are strictly positive, which means that the total fare is given by $B^{\prime}(Q ; \sigma)$ in the high-demand state. Because carrier services are perfectly complementary, it can also not be optimal for a carrier to invest into larger vehicles than the other carrier. This is because some of the costly capacity would again be unused. In equilibrium, it therefore holds that $k_{1}=k_{2}$. Anticipating this result, quantities in the high state of the demand, $Q$, can be written as $k_{1}$ or $k_{2}$. One question is how the total fare $B^{\prime}\left(k_{i} ; \sigma\right)$ translates into individual fares. In principle, all non-negative individual fares that ensure that the sum of individual fares exactly sum up to $B^{\prime}\left(k_{i} ; \sigma\right)$ satisfy the condition of a Nash equilibrium when the capacity constraint is binding in equilibrium: The sum of fares cannot be higher than $B^{\prime}\left(k_{i} ; \sigma\right)$ in equilibrium because this would mean that the capacity constraint is not binding in the high state of demand. The sum of fares can also not be lower than $B^{\prime}\left(k_{i} ; \sigma\right)$ because carriers could sell the same quantities at a higher fare in such situation. However, in a symmetric environment the equilibrium where the total fare is evenly split up between carriers may be considered as a focal point. Therefore, as a starting point, we impose:

Assumption 1 Carriers evenly share the total fare in the high state of demand so that $p_{1}=p_{2}=B^{\prime}\left(k_{i} ; \sigma\right) k_{i} / 2-g\left(k_{i}\right)$ when $\varepsilon=\sigma$.

As will be discussed below, the assumption of an even split of revenues in the high state of demand, will be important for some of our results. We use Assumption 1 to write individual profits in the high state of demand as $B^{\prime}\left(k_{i} ; \sigma\right) k_{i} / 2-g\left(k_{i}\right)$, which helps to derive first insights about equilibrium capacity choices. The capacity constraint is binding in the high-demand state only if, in the pricing stage, it is ensured that carriers cannot increase profit by an individual increase of the own fare. Without loss of generality, 
consider carrier 1 and assume that carrier 2's fare is given by $B^{\prime}(q ; \sigma) / 2$ with $q=k_{1}=k_{2}$. A marginal reduction in the quantity $q$ can be achieved by an increase in the own fare. The corresponding increase in 1's profit can be written as $-B^{\prime}\left(k_{i} ; \sigma\right) / 2-k_{i} B^{\prime \prime}\left(k_{i} ; \sigma\right)$ for $q=k_{i}$ (which abstracts from capacity costs because quantities are reduced while capacities are fixed in the pricing stage). Thus, there is an upper limit for capacities $k_{i}$, denoted $\bar{k}$, implicitly determined by the condition $-B^{\prime}\left(k_{i} ; \sigma\right) / 2-k_{i} B^{\prime \prime}\left(k_{i} ; \sigma\right)=0$, which ensures that for any capacity less than the upper limit $\bar{k}$, the capacity constraint is binding in the pricing stage for $\varepsilon=\sigma$ (i.e., in the high state of demand). The condition that $k_{i} \leq \bar{k}$ is henceforth called the regime constraint. Anticipating that the capacity constraint is binding in the high state of demand and that fares $p_{1}$ and $p_{2}$ are the same in equilibrium, expected individual profits, denoted $E \pi_{i}$, can be written as

$$
E \pi_{i}=\frac{1}{2}\left(p_{i} D(\eta ;-\sigma)+\frac{B^{\prime}\left(k_{i} ; \sigma\right)}{2} k_{i}\right)-g\left(k_{i}\right) .
$$

There is a continuum of symmetric equilibrium capacities; for example, zero capacities are an equilibrium outcome because given that one carrier chooses zero capacity, the other carrier will also choose zero capacity. But, with the assumption that carriers do not expect the other carrier's capacity to be strictly binding, there is only one equilibrium candidate, which satisfies the first-order condition $\partial E \pi_{i} / \partial k_{i}=0$. If both carriers would choose capacities accordingly, the carriers' beliefs that the other capacity is not strictly binding, is indeed fulfilled because capacity is just binding in this scenario, where "just" means that capacity choice is an interior and a corner solution at the same time (therefore, the capacity constraint is binding but not strictly binding). We concentrate on this candidate, as it is the most efficient in the sense of Pareto superiority among the set of candidates for equilibrium capacity choices and therefore keeps our assessments of the efficiency gain from merging, and the efficiency loss from non-coordination, conservative. We can write the first-order condition for capacity choices as $\left(k_{i} B^{\prime \prime}\left(k_{i} ; \sigma\right)+B^{\prime}\left(k_{i} ; \sigma\right)\right) / 4-g^{\prime}=0$, where the first term on the left-hand side is evaluated by $1 / 4$ because it refers to the high state of demand, which occurs with a probability of one half, and because the total fare is evenly divided between carriers in the high state of demand. ${ }^{8}$ Comparing this condition with the condition $B^{\prime}\left(k_{i} ; \sigma\right) / 2+k_{i} B^{\prime \prime}\left(k_{i} ; \sigma\right)=0$ that implicitly determines the upper limit for

\footnotetext{
${ }^{8}$ Again, the second-order conditions are satisfied if the marginal benefit function is sufficiently concave (Czerny, Verhoef and Zhang, 2015). The consideration of an interior solution can further be justified by the fact that carriers will typically also serve some non-stop passengers in their home market, which may ensure the existence of interior solutions.
} 


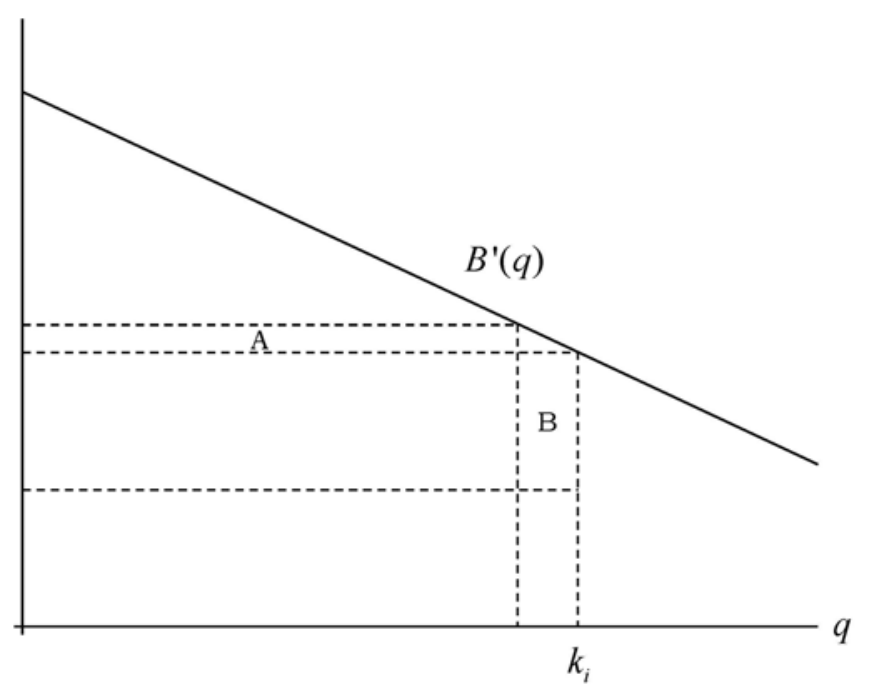

Figure 1: Regime constraint binding when capacities are sufficiently large $(\mathrm{A}>\mathrm{B})$.

equilibrium capacity $\bar{k}$, reveals:

Lemma 1 If marginal capacity cost, $g^{\prime}$, are sufficiently high in the sense that they exceed $-\left(3 k_{i} B^{\prime \prime}\left(k_{i} ; \sigma\right)+B^{\prime}\left(k_{i} ; \sigma\right)\right) / 4$ in equilibrium, the regime constraint is not binding and equilibrium capacities are smaller than the upper limit $\bar{k}$, while the regime constraint is binding and equilibrium capacities are equal to the upper limit $\bar{k}$, otherwise.

Thus, for high enough marginal capacity costs, carrier capacities are so small in equilibrium that the regime constraint is not binding. If, on the other hand, marginal capacity costs are low, this tends to increase equilibrium capacities so that the regime constraint becomes binding. The regime constraint therefore is useful to distinguish between regimes with high and low marginal capacity costs. Figures 1 and 2 illustrate these cases. Figure 1 assumes large capacities relative to Figure 2. In both Figures 1 and 2, a unilateral increase in fare reduces aggregate revenue. However, the firm that increases the fare gains by increasing its share in revenues. The overall individual gain from a unilateral increase in the fare is given by the difference between areas A and B. This difference is positive when capacities are large and negative when capacities are small.

Consider the case where the regime constraint is not binding. In this case, the firstorder conditions, $\partial E \pi_{i} / \partial k_{i}=0$, imply that the carriers' incentives to invest in capacity are reduced because carriers receive only a share of one half of the revenues in the high demand state. But, note that carriers also evenly share capacity costs because both carriers have to 


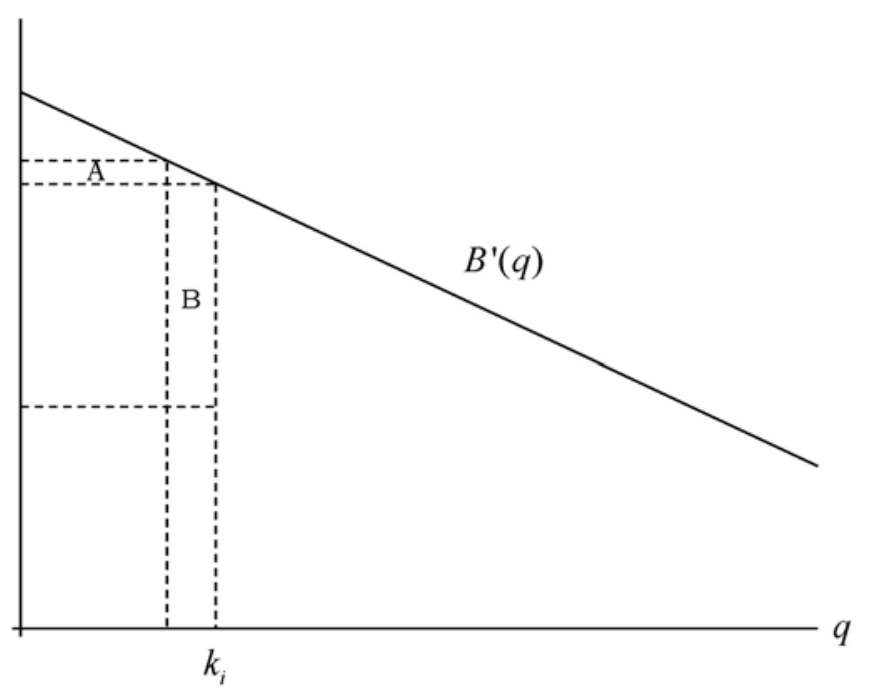

Figure 2: Regime constraint not binding when capacities are sufficiently small $(\mathrm{A}<\mathrm{B})$.

increase capacity in order to be able to increase passenger volume. Thus, the independent carriers' incentives to invest in vehicle size are not different from the alliance carriers' incentives when marginal capacity costs are sufficiently high. This is because alliance carriers take into account the entire increase in revenues but also the entire increase in capacity costs on the two connections. Consider the case where the regime constraint is binding. In this case, the alliance carriers' incentive structures are essentially the same as in the previous case, while for independent carriers the incentives to invest in capacity are reduced. This leads to:

Proposition 1 Capacities are the same for independent and alliance carriers when the regime constraint is not binding as specified in Lemma 1, while carrier collaboration increases capacities relative to independent carriers, otherwise.

That collaboration can increase capacity relative to independent carriers is the result of a special form of double marginalization in capacity that occurs when carriers are independent. Recall that carriers reduce capacity to $\bar{k}$ because carriers have an incentive to increase markups while ignoring the effect of their increase in markups on the other carrier's profit. This shows how double marginalization can enter the picture in the high state of demand. A similar result - centralization can lead to an increase of capacity when firms' produce complementary products - has been derived by Netessine and Zhang (2005). 
Their analysis, however, assumes that retail prices are exogenously given. Furthermore, they model complementarity in a more ad hoc fashion assuming that a retailer's demand is increasing in the other carriers demand. In our case, a carrier's demand is increasing in the other carrier's output only if the capacity constraint is binding. ${ }^{9}$

The result presented in Proposition 1 that capacities can be the same with independent and alliance carriers does not depend on the structure of the stochastic process (Bernoulli distribution of $\varepsilon$ ) but only on the fact that revenue shares are equal to the cost shares. Similar results can also obtained when carrier costs differ. For instance, assume that land costs are higher for carrier 1 than for carrier 2 and denote individual carrier costs as $g_{i}$ with $g_{i}=g_{i}\left(k_{i}\right)$ and $g_{1}^{\prime}\left(k_{1}\right)>g_{2}^{\prime}\left(k_{2}\right)$ for $k_{1}=k_{2}$. In this situation, capacity investments are the same for independent and alliance carriers if revenue shares are determined by the fraction of marginal capacity costs $g_{1}^{\prime}\left(k_{1}\right) /\left(g_{1}^{\prime}\left(k_{1}\right)+g_{2}^{\prime}\left(k_{2}\right)\right)$. In the case of asymmetric capacity costs, the sharing of revenues according to the fraction of marginal capacity costs may indeed be considered as a focal point because it leads to asymmetric revenue shares and ensures that the carrier with the higher marginal cost receives a higher share of revenues. If revenues are not shared according to the marginal fraction of marginal capacity costs, then carrier collaboration can have a positive effect on capacity even when the regime constraint is not binding. This is because the carriers' incentives to invest in capacity are positively related to the own share in the total fare, which means that the overall capacity is determined by investments of the carrier that receives the small share relative to the fraction of marginal capacity costs when carriers are independent.

Consider the case where the regime constraint is not binding, again (when Assumption 1 is true and carriers charge the same fares in the high state of demand): Carrier collaboration reduces fares in the low state of demand but does not change capacity. Letting $E l_{i}$ with $E l_{i}=\left(D(\eta ;-\sigma)+k_{i}\right) /\left(2 k_{i}\right)$ denote the expected load factor, this leads to:

Proposition 2 Alliance carriers increase expected load factors relative to independent carriers when (i) capacity is one-dimensional in the sense that carriers can only vary vehicle sizes (and vehicle numbers are fixed) and (ii) the regime constraint is not binding.

This result is in line with the empirical findings from previous studies that showed that

\footnotetext{
${ }^{9}$ Netessine and Shumsky (2005) use a similar framework (for example, they consider exogenous air fares) as the one developed by Nestessine and Zhang (2005) to analyze airline booking limits for low and high fare classes. They find that the effect of centralization on booking limits is highly ambiguous when networks are complementary.
} 
carrier collaboration can increase load factors. However, the effect of carrier collaboration on load factors is more difficult to derive when the regime constraint is binding. This is because equilibrium quantities are increased by carrier collaboration but also capacity is increased by carrier collaboration. The following example shows that collaboration can increase load factors also when the regime constraint is binding.

Example 1 Benefits are quadratic and of the form $B=(a+b \varepsilon) q-b q^{2} / 2$ with $a, b>$ 0. ${ }^{10}$ Capacity costs are $g=w k_{i}^{2} / 2$. Consider independent carriers. In the low state of demand, the total fare is $p_{1}+p_{2}=2(a-b \sigma) / 3$ and the passenger quantity is $q=$ $(a-b \sigma) /(3 b)$ for $\sigma<a / b$. Capacities are $k_{i}=\bar{k}=(a+b \sigma) /(3 b)$ for $w<b / 4$ and $k_{i}=(a+b \sigma) /(2(b+2 w))$ for $w \geq b / 4$. Altogether this leads to an expected load factor $E l=a /(a+b \sigma)$ for low capacity costs in the sense that $w<b / 4$, where the regime constraint is binding. Consider alliance carriers. In the low state of demand, the total fare is $p_{1}+p_{2}=(a-b \sigma) / 2$ and the passenger quantity is $q=(a-b \sigma) /(2 b)$. Capacity is $k_{i}=$ $(a+b \sigma) /(2(b+2 w))$. Expected load factor is $E l=a /(a+b \sigma)+w(a-b \sigma) /(b(a+b \sigma))$. Comparison with expected load factors of independent carriers for $w<b / 4$ reveals that load factors can be increased by carrier collaboration independent of whether the regime constraint is binding or not binding (recall that expected load factors increase for $w>b / 4$ by Proposition 2). The capacity cost parameter, w, must be sufficiently low to ensure that the capacity constraint is not binding in the low state of demand. More specifically, to ensure that expected load factors are less than one-hundred percent in the alliance carrier scenario, $w<b^{2} \sigma /(a-b \sigma)(\Leftrightarrow \sigma>a w /(b+w))$ must be satisfied.

It is possible to derive the welfare effects of carrier collaboration under the conditions considered in this part of the paper, i.e., with fixed vehicle numbers, where welfare is the difference between benefit $B$ and total capacity costs $g\left(k_{1}\right)+g\left(k_{2}\right)$. Expected welfare is the sum of expected consumer and expected producer surplus. Furthermore, expected total profit, $E \pi_{1}+E \pi_{2}$, unambiguously increases by carrier collaboration. This is because collaborating carriers can easily replicate the behavior of independent firms, and if they deviate, this means that aggregate expected profit is increased by collaboration. Furthermore, collaborating carriers deviate from the behavior of independent firms by reducing fares in the low state of demand, while behavior is unchanged in the high state of the

\footnotetext{
${ }^{10}$ The multiplication of the noise term with the demand slope parameter, $b$, means that errors are added to the demand function, i.e., demand is separable into two additive parts, $D=\bar{D}+\varepsilon$.
} 
demand when the total fare is evenly shared between carriers in the high state of demand or increases capacity investments when the total fare is not evenly shared between carriers or the regime constraint is binding in the high state of demand. Thus, consumer surplus, $B-\left(p_{1}+p_{2}\right) \cdot k_{i}$, in the low and high states of demand increases with alliance carriers relative to independent carriers. Thus, collaboration increases expected welfare.

A feature of the present study is that capacity and load factors are endogenous, and this is relevant for the evaluation of alliances for both firms and policy makers. The evaluation of carrier collaboration can be too optimistic if load factors are considered as fixed. This is because the positive effects of carrier collaboration on revenue due to the elimination of double marginalization, may only exist in periods of low demand (when only vehicle sizes are endogenous). Thus, in periods of high demand, the effects of carrier collaboration on revenue may be overestimated if one abstracts away from the fact that the organizational structure, i.e., whether carriers are independent or form an alliance, may not change market outcomes in the high state of demand. A similar line of reasoning can be constructed for welfare. More specifically, this means that the welfare effects of carrier collaboration may be overestimated when load factors are considered as fixed.

\section{Vehicle Sizes and Vehicle Numbers Endogenous}

While the previous section treated carrier capacity as one-dimensional, i.e., fleet sizes could only be changed by changes in vehicle sizes, this section considers vehicle sizes and vehicle numbers as endogenous. This is to derive insights on the equilibrium fleet size and structure when carrier networks are complementary.

To analyze this, assume that the carriers' capacities are determined by the product of the number of vehicles, denoted $f_{i}$, and the size of vehicles, denoted $s_{i}$, which implies $k_{i}=s_{i} f_{i}$. Costs are still a convex function of capacity $k_{i}\left(=s_{i} f_{i}\right)$. In addition, and to ensure the existence of solutions, there is a given cost, denoted $d$ with $d>0$, per unit of $f_{i} .{ }^{11}$ This leads to (strictly convex) cost $d f_{i}+g\left(s_{i} f_{i}\right)$. Another feature is that a carrier's service quality can be related to $f_{i}$. This is true because passengers are more likely to travel at their preferred time, which reduces so called schedule delay costs, when more vehicles are in operation (e.g., Douglas and Miller, 1974). To capture this, assume

\footnotetext{
${ }^{11}$ For $d=0$, it is optimal to choose an arbitrarily small seat capacity and increase frequency supply to infinity because frequency supply increases demand, which is not true for seat capacity (Czerny, Verhoef and Zhang, 2015).
} 
that schedule delay costs, denoted $\Gamma$, are decreasing in frequencies and a strictly convex function of frequencies $f_{1}$ and $f_{2}$, i.e., $\Gamma=\Gamma\left(f_{1}, f_{2}\right)$ with $\partial \Gamma / \partial f_{i}<0, \partial^{2} \Gamma / \partial f_{i}^{2}>0$ and $\left|\partial^{2} \Gamma / \partial f_{1} \partial f_{2}\right|<\sqrt{\partial^{2} \Gamma / \partial f_{1}^{2} \cdot \partial^{2} \Gamma / \partial f_{2}^{2}}$. Thus, frequencies can be substitutes (i.e., $\left.\partial^{2} \Gamma / \partial f_{1} \partial f_{2} \geq 0\right)$ or complements (i.e., $\left.\partial^{2} \Gamma / \partial f_{1} \partial f_{2}<0\right)$ from the passengers' viewpoint. In the case of substitutes, overall schedule delays may be unchanged if one carrier increases frequency and the other carrier reduces frequency. In the case of complements, an increase in one carrier's frequencies becomes more effective if the other carrier also increases frequencies. One reason may be that a joint increase in frequencies facilitates schedule coordination. This leads to the generalized price of traveling denoted as $\psi$ with $\psi=\eta+\Gamma\left(f_{1}, f_{2}\right)$.

With one-dimensional capacity, the pricing decisions in the low-demand state and the capacity decisions, which are only relevant in the high-demand state, are separated in the sense that the sequential structure of the decisions is irrelevant for the result. This changes in the current scenario with two-dimensional capacity, where the decision about the frequencies $f_{i}$ affects fares in the low state of the demand and both capacities as well as fares in the high state of demand.

Second stage. Consider the low state of demand. The carriers' best responses in terms of fares are implicitly determined by the first-order conditions $\partial \pi_{i} / \partial p_{i}=D(\psi ;-\sigma)+$ $p_{i} D^{\prime}(\psi ;-\sigma)=0$ when carriers are independent. As before, with one-dimensional capacity, this leads to double marginalization, which can be eliminated by carrier collaboration. However, because the current version of the model assumes that service quality in terms of frequency supply is endogenous, it is useful to understand how changes in frequencies affect equilibrium fares in the second stage. Given that (standard) assumptions on the convexity of demand, $D$, are satisfied, totally differentiating these first-order conditions, $\partial \pi_{i} / \partial p_{i}=0$, with respect to fares and frequency $f_{i}$ reveals:

Lemma 2 Consider independent carriers in the low state of demand: An increase in own frequency $f_{i}$ increases the own equilibrium fare $p_{i}$ (i.e., $\partial p_{i} / \partial f_{i}>0$ ) and the other carrier's equilibrium fare $p_{j}$ (i.e., $\partial p_{j} / \partial f_{i}>0$ ) but reduces the generalized price $\psi$ (i.e., $\left.\partial \psi / \partial f_{i}<0\right)$.

This result can be explained as follows. Fares are strategic substitutes in the sense of Bulow, Geanakoplos and Klemperer (1985), which means that the other carrier's best 
response in terms of fares is a decreasing function of the own fare. ${ }^{12}$ Since an increase in one carrier's frequency increases the own fare but reduces the generalized price of traveling (because the reduction in schedule delays dominates the increase in the own fare), this leads to an increase in the other carrier's fare as well.

Consider the high state of demand. The capacity constraint is assumed to be binding in the high state of the demand leading to the total fare $B^{\prime}\left(s_{i} f_{i} ; \sigma\right)-\Gamma\left(f_{1}, f_{2}\right)$. For the sake of a conservative assessment of collaboration strategies, we further assume that carriers evenly share the total fare in the high state of demand, i.e., $p_{1}=p_{2}=$ $\left(B^{\prime}\left(s_{i} f_{i} ; \sigma\right)-\Gamma\left(f_{1}, f_{2}\right)\right) / 2$. This is analogue to Assumption 1. With endogenous vehicle numbers the upper limit for equilibrium capacity, $\bar{k}$, is implicitly determined by the condition $-\left(B^{\prime}\left(k_{i} ; \sigma\right)-\Gamma\left(f_{1}, f_{2}\right)\right) / 2-k_{i} B^{\prime \prime}\left(k_{i} ; \sigma\right)=0$.

First stage. For the same reasons as discussed in the previous chapter with one-dimensional capacity, capacities $s_{1} f_{1}$ and $s_{2} f_{2}$ are the same in equilibrium. Expected profits can then be written as ${ }^{13}$

$$
E \pi_{i}=\frac{1}{2}\left(p_{i} D(\psi ;-\sigma)+\frac{B^{\prime}\left(s_{i} f_{i} ; \sigma\right)-\Gamma\left(f_{1}, f_{2}\right)}{2} s_{i} f_{i}\right)-d f_{i}-g\left(s_{i} f_{i}\right)
$$

For the sake of conservativeness, consider (interior) best responses in terms of seat capacities in the sense that best responses are implicitly determined by the first-order conditions $\partial E \pi_{i} / \partial s_{i}=0$, which can be written as

$$
\left(\frac{1}{4}\left(B^{\prime \prime}\left(s_{i} f_{i} ; \sigma\right) s_{i} f_{i}+B^{\prime}\left(s_{i} f_{i} ; \sigma\right)-\Gamma\left(f_{1}, f_{2}\right)\right)-g^{\prime}\left(s_{i} f_{i}\right)\right) f_{i}=0
$$

Another way of writing this first-order condition is $\partial E \pi_{i} / \partial k_{i} \cdot \partial k_{i} / \partial s_{i}=0$. This shows that the first-order condition (3) is equivalent to $\partial E \pi_{i} / \partial k_{i}=0$, which means that seat capacities are (only) used to optimize capacities $k_{i}$. This condition implies that the regime constraint is binding if the marginal capacity costs are low in the sense that $g^{\prime}<-\left(3 k_{i} B^{\prime \prime}\left(k_{i} ; \sigma\right)+B^{\prime}\left(k_{i} ; \sigma\right)-\Gamma\left(f_{1}, f_{2}\right)\right) / 4$

Similarly to before, where capacity could be varied only by a change in vehicle size, the individual carrier's incentives to invest into capacity are the same for independent and

\footnotetext{
${ }^{12}$ Fares are strategic substitutes only if the demand function is sufficiently concave, which is a maintained assumption in the present study.

${ }^{13}$ One may wonder whether negotiation power may be related to frequency supply in the sense that the carrier offering higher frequency receives a higher revenue share. While this may be true, capturing this effect would complicate the analysis. We believe that our main results are largely independent of the assumption that revenues are shared evenly.
} 
alliance carriers when frequencies are given and the same for carriers 1 and 2 (and the total fare is evenly shared in the high state of demand) and the regime constraint is nonbinding. If frequency supplies change, the effects on the incentives to invest in capacity can be described as follows: ${ }^{14}$

Lemma 3 Assume a non-binding regime constraint. Equilibrium capacities, $k_{i}=s_{i} f_{i}$, are increasing in joint frequency supply.

An increase in frequencies reduces schedule delays and increases demand because this reduces generalized prices in the second stage by Lemma 2. Intuitively, such an increase in demand increases the incentives to invest in capacity as pointed out in Lemma 3. The following analysis shows indeed that the incentives to invest in frequencies are higher for alliance carriers relative to independent carriers, which is true for the cases of binding and non-binding regime constraints.

The choice of frequency is more complex compared to the choice of vehicle sizes because frequency affects both capacity and demand. To see this, assume that the regime constraint is non-binding and write the first-order condition for an interior solution for equilibrium seat capacity and frequency supply, which anticipates the second-stage pricing behavior, as $\partial E \pi_{i} / \partial k_{i} \cdot \partial k_{i} / \partial f_{i}+\partial E \pi_{i} / \partial \Gamma \cdot \partial \Gamma / \partial f_{i}+\partial E \pi_{i} / \partial p_{j} \cdot \partial p_{j} / \partial f_{i}=0$. The left-hand side shows that frequency is also chosen to optimize capacity (the first term on the left-hand side), and in addition it is chosen to optimize service quality in terms of schedule delays, and to strategically manipulate the other carrier's fare in the low-demand state. But, the first term is zero by the choice of seat capacity; thus, only the remaining two effects on schedule delay costs and the strategic manipulation of fares are relevant for frequency choices, and there sum should therefore be equal to zero in optimum. Since capacity is optimized by the choice of seat capacity, the same is true when the regime constraint is binding; so the rationale for frequency supply does not depend on whether the regime constraint is binding or non-binding.

Using expected profit in (2), best responses in terms of frequencies are implicitly determined by the first-order conditions $\partial E \pi_{i} / \partial f_{i}=0$, which can be written as

$$
-\frac{1}{2}\left(D^{\prime}(\psi ;-\sigma) \frac{\partial p_{j}}{\partial f_{i}}+\left(D(\psi ;-\sigma)+\frac{s_{i} f_{i}}{2}\right) \frac{\partial \Gamma}{\partial f_{i}}\right)-d-g^{\prime}\left(s_{i} f_{i}\right) s_{i}=0 .
$$

\footnotetext{
${ }^{14}$ The following lemma assumes that standard assumptions on the convexity of the inverse demand function, $B^{\prime}$, are satisfied.
} 
The left-hand side can be used to show that the independent carriers' choice of frequency is too low from the collaborating carriers' viewpoint for two reasons. First, the reduction in schedule delay costs achieved in the high state of demand, $s_{i} f_{i} \cdot \partial \Gamma / \partial f_{i}$, is evaluated by one half because the total fare is divided evenly between carriers 1 and 2 in the high state of demand. ${ }^{15}$ Second, carriers anticipate that an increase in own frequency increases the other carrier's fare in the low state of demand because $\partial p_{j} / \partial f_{i}>0$ by Lemma 2 . This means that carriers strategically reduce frequency supply in the first stage in order to lower the other carrier's equilibrium fare (in the low state of demand). Both of these effects can be eliminated by carrier collaboration. If functional forms for capacity and schedule delay cost functions $g$ and $\Gamma$, respectively, are sufficiently convex, this implies:

Proposition 3 Alliance carriers increase fleet capacities $k_{i}\left(=s_{i} f_{i}\right)$ and frequency supplies $f_{i}$ relative to independent carriers when vehicle numbers are endogenously determined.

While capacity is increased by carrier collaboration, the effect of carrier collaboration on expected load factors are difficult to predict when both vehicle sizes and vehicle numbers are endogenous. This is because an increase in frequency supply, thus, service quality increases both capacity and demand. The following numerical examples illustrate that expected load factors can be substantially increased by carrier collaboration.

Example 2 Assume quadratic benefits and costs with $a=2, b=1$ and $d=1 / 20$. The schedule delays are given be the sum of schedule delays at each connection, i.e., $\Gamma=\left(1 / f_{1}+1 / f_{2}\right) / 2 .{ }^{16}$ The latter captures that frequencies can be substitutes from the passengers' viewpoint. Figure 3 depicts equilibrium capacities of independent carriers for low (dashed line) and high (solid line) values of the standard deviation parameter where $\sigma=1 / 4$ and $\sigma=1 / 2$, respectively. The range of the capacity cost parameter, $w$, is chosen to ensure that expected load factors are below one-hundred percent (dashed line) and that

\footnotetext{
${ }^{15}$ A similar effect would also occur if the total fare is divided according to an arbitrary share between carriers.

${ }^{16}$ Brueckner (2004) uses similar functional forms for schedule delay costs on a single connection. Silva, Verhoef and v. d. Berg (2014) assume that the overall schedule delays are given be the sum of schedule delays at each connection, which implies that frequencies are substitutes. A more general approach is to describe schedule delay costs $\Gamma$ by a CES function $F$ of the form $F=F\left(\left(f_{1}^{x}+f_{2}^{x}\right)^{1 / x}\right)$, while we consider the special case $x=1$ in the example.
} 


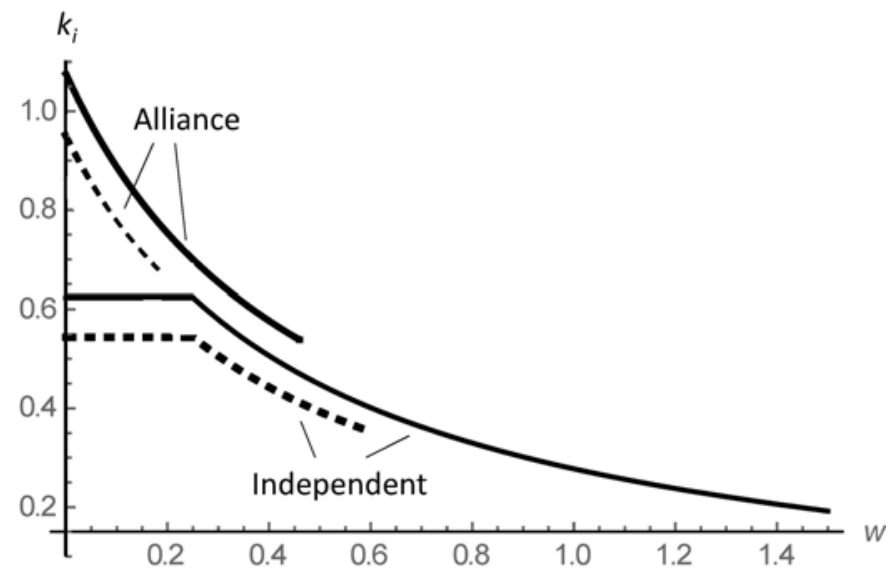

Figure 3: Capacities for low and high uncertainty in the sense that $\sigma=1 / 4$ (dashed line) and $\sigma=1 / 2$ (solid line), respectively, depending on capacity cost parameter $w$. Parameters: $a=2, b=1, d=1 / 20$.

expected profits are non-negative (solid line). The figure shows that equilibrium fleet capacities increase in uncertainty measured by the uncertainty parameter, which is independent of whether the regime constraint is binding or non-binding. Furthermore, equilibrium fleet capacities are constant in the capacity cost parameter as long as it is less than $1 / 4$ because the regime constraint is binding in these cases. For sufficiently high values of the capacity cost parameter, the equilibrium fleet capacities are decreasing in the capacity cost parameter, and this reduction in equilibrium fleet capacities increases expected load factors as shown by Figure 4. For the relevant parameter instances, fleet capacities and expected load factors are increased by alliance carriers relative to independent carriers.

Using symmetry, welfare depending on vehicle sizes and vehicle numbers can be written as $B-\left(\min \left\{q, s_{i} f_{i}\right\} \cdot \Gamma+2\left(d f_{i}+g\left(s_{i} f_{i}\right)\right)\right)$. To understand that carrier collaboration unambiguously increases welfare when both vehicle sizes and vehicle numbers are endogenous, it is useful to understand that a monopoly carrier can perfectly internalize any reduction in schedule delay costs by an increase in fares when schedule delay costs are the same for all passengers (Czerny and Zhang, 2015). This is because the generalized price of traveling, $\psi$, is the same for all passengers; thus, a reduction in schedule delay costs can be perfectly internalized by a corresponding increase in fares. More specifically, collaborating carriers can perfectly internalize the reduction in schedule delay costs in the 


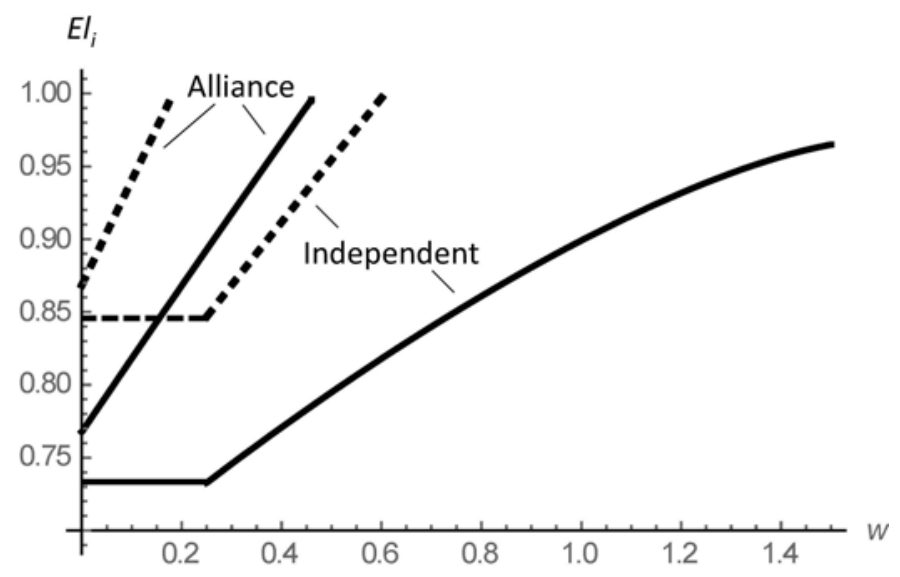

Figure 4: Expected load factors for low and high uncertainty in the sense that $\sigma=1 / 4$ (dashed line) and $\sigma=1 / 2$ (solid line), respectively, depending on capacity cost parameter $w$. Parameters: $a=2, b=1, d=1 / 20$.

high state of demand, $s_{i} f_{i} \cdot \partial \Gamma / \partial f_{i}$, and will not choose frequencies strategically in order to manipulate the other carrier's fare, which means that collaborating carriers abstract away from $\partial p_{j} / \partial f_{i}>0$. Thus, if collaborating carriers increase frequencies, this is in line with the behavior of a social optimizer (for given passenger quantities). Furthermore, since capacities, $s_{i} f_{i}$, are increased by carrier collaboration, this means that the generalized price is reduced in the high state of demand, which increases consumer surplus, $B-q \psi$, in the high state of demand. Altogether, this means that alliance carriers increase welfare relative to independent carriers and that is independent of whether vehicle numbers are considered as fixed or variable. This strengthens the case of antitrust exemption when networks are perfectly complementarity.

\section{Conclusions}

Our study indicates that load factors can be valuable predictors for the financial and social performance of carriers. This is true in the sense that carrier collaboration increases load factors of the carriers involved, profits and social welfare when networks are perfectly complementary. If a given number of vehicles can be varied only in size, this is for two reasons. First, fleet sizes can be independent of collaboration when carriers share revenues evenly and there is a binding capacity constraint and marginal seat costs are sufficiently 
high. Second, because carrier collaboration can eliminate double marginalization in fares and further eliminate double marginalization in the choice of fleet sizes when the marginal seat costs are sufficiently low.

If both vehicle sizes and vehicle numbers can be changed by carriers, collaboration increases fleet sizes (given by the product of the number and the size of vehicles) and vehicle numbers. The number of vehicles increases because alliance members recognize that increasing the own service quality in terms of frequency supply increases own profit but also the profit of their partners. Fleet size increases because (i) an increase in frequency increases demand, which increases the incentives to invest into fleet size and (ii) double marginalization in terms of capacity choices can be avoided when the marginal costs of fleet capacity expansion are sufficiently low. However, the effects of collaboration on vehicle sizes, thus, also load factors are hard to predict when both vehicle sizes and vehicle numbers are endogenous because carrier collaboration increases both passenger quantities and fleet sizes. Numerical simulations showed that collaboration can lead to an increase in expected load factors also in these situations. In all the scenarios we considered, carrier collaboration increases social welfare because it reduces generalized prices through reductions in fares and schedule delays.

The analysis provides several avenues for future research. First off, we concentrated on perfectly complementary networks, while carrier networks can be overlapping in practice. ${ }^{17}$ Airline networks are a prominent example. International airline markets are typically served in a hub-and-spoke fashion, which means that some passengers have to change aircraft at the hub to reach their final destination. In such situations, airlines often serve hub-to-hub connections in parallel, and policy makers are concerned that carrier collaboration will lead to collusive pricing and higher fares on the parallel parts of the networks, which changes the welfare evaluation. ${ }^{18}$ Another issue that changes the welfare evaluation is the existence of passenger types with distinct time valuations (Czerny and Zhang, 2011, 2014 and forthcoming). More specifically, the present analysis abstracts away from the existence of business passengers who possibly attach a higher time valuation to schedule delays relative to leisure passengers. With passenger types, profit maximizing carriers are concerned with marginal time valuations attached to schedule delays (i.e., average time valuation of incremental passengers) and not average time valuations, where

\footnotetext{
${ }^{17}$ See Zhang and Czerny (2012) for a discussion of the relevant literature on overlapping airline networks.

${ }^{18}$ For example, Zhang and Czerny (2012) mention that carrier collaboration may also lead to market exit of one partner airline on the hub-to-hub part and thus change carrier networks.
} 
the latter are of concern to a social optimizer (e.g., Czerny and Zhang, 2014 and 2015). Furthermore, business passengers exert less elastic demand relative to leisure passengers, which implies that marginal time valuations are smaller than average time valuations. Altogether this means that the collaborating carriers' incentives for frequency supply can be too low from the social viewpoint, which further complicates the welfare analysis of carrier collaboration. Finally, it would be useful to empirically validate the theoretical insights derived in this paper. 


\section{References}

Adler, N. and Hanany, E. (2015), Regulating inter-firm agreements: The case of airline codesharing in parallel networks, unpublished.

Aguirre, I., Cowan, S. and Vickers, J. (2010), Monopoly price discrimination and demand curvature, American Economic Review 100: 1601-1615.

Armantier, O. and Richard, O. (2008), Domestic airline alliances and consumer welfare, RAND Journal of Economics 39: 875-904.

Bailey, E.E., Graham, D.R., Kaplan, D.P. (1985), Deregulating the Airlines. MIT Press, Cambridge, MA.

Bamberger, G. E., Carlton, D. W. and Neumann, L. R. (2004), An empirical investigation of the competitive effects of domestic airline alliances, Journal of Law and Economics 47: $195-222$.

Behn, B. K. and Riley, R. A. Jr. (1999), Using nonfinancial information to predict financial performance: the case of the U.S. airline industry, Journal of Accounting, Auditing and Finance 14: 29-56.

Brueckner, J. K. (2001), The economics of international codesharing: an analysis of airline alliances, International Journal of Industrial Organization 19: 1475-1498.

Brueckner, J. K. (2004), Network structure and airline scheduling, Journal of Industrial Economics 52: 291-312.

Brueckner, J. K. and Whalen, T. W. (2000), The price effects of international alliances, Journal of Law and Economics 43: 503-545.

Brueckner, J. K. and Zhang, A. (2010), Airline emission charges: Effects on airfares, service quality, and aircraft design, Transportation Research Part B 44: 960-971.

Bulow, J. I., Geanakoplos, J. D. and Klemperer, P. D. (1985), Multimarket oligopoly: Strategic substitutes and complements, Journal of Political Economy 93: 488-511.

Chen, F. C.-Y. and Chen, C. (2003), The effects of strategic alliances and risk pooling on the load factors of international airline operations, Transportation Research Part E 39: $19-34$. 
Czerny, A. I. (2009), Code-sharing, price discrimination and welfare losses, Journal of Transport Economics and Policy 43: 193-210.

Czerny, A. I. (forthcoming), The role of capital costs for airline responses to emission charges, Journal of Transport Economics and Policy.

Czerny, A. I., Verhoef, E. T. and Zhang, A. (2015), A theory of continuous uncertainty types, Tinbergen Institute Discussion Paper TI 2015-065/VIII.

Czerny, A. I. and Zhang, A. (2011), Airport congestion pricing and passenger types, Transportation Research Part B 45: 595-604.

Czerny, A. I. and Zhang, A. (2014), Airport peak-load pricing revisited: The case of peak and uniform tolls, Economics of Transportation 3: 90-101.

Czerny, A. I. and Zhang, A. (2014), Airport congestion pricing when airlines price discriminate, Transportation Research Part B 65: 77-89.

Czerny, A. I. and Zhang, A. (forthcoming), Third-degree price discrimination in the presence of congestion externality, Canadian Journal of Economics.

Czerny, A. I. and Zhang, A. (2015), How to mix per-flight and per-passenger based airport charges, Transportation Research Part A 71: 77-95.

Devrient, L., Burghouwt, G., Derudder, B., de Wit, J., Witlox, F. (2009): Calculating load factors for the transatlantic airline market using supply and demand data - A note on the identification of gaps in the available airline statistics, Journal of Air Transport Management 15: 337-343.

Douglas, G. W. and Miller, J. C. (1974), Quality competition, industry equilibrium, and efficiency in the price-constrained airline market, American Economic Review 64: 657-669.

Economides, N. and Salop, S. C. (1992), Competition and integration among complements, and network market structure, Journal of Industrial Economics 40: 105-123.

Graham, D. R., Kaplan, D. P. and Sibley, D. S. (1983), Efficiency and competition in the airline industry, Bell Journal of Economics 14: 118-138.

Hu, Q. (2010), Network game and capacity investment under market uncertainty, Production and Operations Management 19: 98-110. 
Hu, X., Caldentey, R. and Vulcano, G. (2013), Revenue sharing in airline alliances, Management Science 59: 1177-1195.

Iatrou, K. and Alamdari, F. (2005): The empirical analysis of the impact of alliances on airline operations, Journal of Air Transport Management 11: 127-134.

Lazzarini, S. G. (2007), The impact of membership in competing alliance constellations: evidence on the operational performance of global airlines, Strategic Management Journal 28: 345-367.

Li, J. and Netessine, S. (2011), Partnering with competitors - Effects of alliances on airline entry and capacity decisions, INSEAD Working Paper No. 2011/24/TOM.

Miller, J. C. (1972), A time of day model for aircraft scheduling, Transportation Science 6: $221-246$.

Netessine, S. and Shumsky, R. A. (2005), Revenue management games: horizontal and vertical competition, Management Science 51: 813-831.

Netessine, S. and Zhang, F. (2005), Positive vs. negative externalities in inventory management: implications for supply chain design, Manufacturing and Service Operations Management 7: 58-73.

Silva, H., Verhoef, E. T. and van den Berg, V. A. C. (2014), Airline route structure competition and network policy, Transportation Research Part B 47: 320-343.

Spengler, J. J. (1950), Vertical integration and antitrust policy, Journal of Political Economy 58: $347-352$.

Viton, P. A. (1986), Air deregulation revisited: Choice of aircraft, load factors, and marginal-cost fares for domestic air travel, Transportation Research Part A 20A: 361-371.

Zhang, A. and Czerny, A. I. (2012), Airports and airlines economics and policy: An interpretive review of recent research, Economics of Transportation 1:15-34.

Zhang, A. and Zhang, Y. (2006), Rivalry between strategic alliances, International Journal of Industrial Organization 24: 287-301. 


\section{Appendix}

\section{A Proofs}

\section{A.1 Proof of Lemma 2}

Denote the first-order conditions for fares in the low state of demand as

$$
\begin{aligned}
& y_{1}=D(\psi,-\sigma)+p_{1} D^{\prime}(\psi,-\sigma)=0, \\
& y_{2}=D(\psi,-\sigma)+p_{2} D^{\prime}(\psi,-\sigma)=0 .
\end{aligned}
$$

Totally differentiating with respect to fares and carrier 1's frequency yields the system of equations

$$
\begin{aligned}
& d y_{1}=\frac{\partial y_{1}}{\partial p_{1}} d p_{1}+\frac{\partial y_{1}}{\partial p_{2}} d p_{2}+\frac{\partial y_{1}}{\partial f_{1}} d f_{1}=0 \\
& d y_{2}=\frac{\partial y_{2}}{\partial p_{1}} d p_{1}+\frac{\partial y_{1}}{\partial p_{2}} d p_{2}+\frac{\partial y_{2}}{\partial f_{1}} d f_{1}=0
\end{aligned}
$$

In matrix form this can be written as

$$
\left(\begin{array}{cc}
\frac{\partial y_{1}}{\partial p_{1}} & \frac{\partial y_{1}}{\partial p_{2}} \\
\frac{\partial y_{2}}{\partial p_{1}} & \frac{\partial y_{2}}{\partial p_{2}}
\end{array}\right)\left(\begin{array}{c}
d p_{1} \\
d p_{2}
\end{array}\right)=-d f_{1}\left(\begin{array}{c}
\frac{\partial y_{1}}{\partial f_{1}} \\
\frac{\partial y_{2}}{\partial f_{1}}
\end{array}\right)
$$

or, more specifically,

$$
\left(\begin{array}{cc}
2 D^{\prime}+p_{1} D^{\prime \prime} & D^{\prime}+p_{1} D^{\prime \prime} \\
D^{\prime}+p_{2} D^{\prime \prime} & 2 D^{\prime}+p_{2} D^{\prime \prime}
\end{array}\right)\left(\begin{array}{c}
d p_{1} \\
d p_{2}
\end{array}\right)=-d f_{1}\left(\begin{array}{c}
-\left(D^{\prime}+p_{1} D^{\prime \prime}\right) \frac{\partial \Gamma}{\partial f_{1}} \\
-\left(D^{\prime}+p_{2} D^{\prime \prime}\right) \frac{\partial \Gamma}{\partial f_{1}}
\end{array}\right),
$$

where arguments are omitted to economize notation.

It is useful to let $\omega(h(x))$ denote the elasticity of the slope of an arbitrary function $h(x)$ with respect to $x$ with $\omega(h(x))=-x h^{\prime \prime} / h^{\prime}$ for $h^{\prime}<0$ and $\omega(h(x))=x h^{\prime \prime} / h^{\prime}$ for $h^{\prime}>0$. This elasticity can be used as a measure for the convexity of function $h .{ }^{19}$ For example, if $\omega(h(x))>0$, function $h$ is strictly convex in $x$, while it is strictly concave if $\omega(h(x))<0$ and linear if $\omega(h(x))=0$. Furthermore, denote the determinant of the first matrix on the left-hand side as $\Psi$ with

$$
\Psi=\operatorname{det}\left(\begin{array}{cc}
2 D^{\prime}+p_{1} D^{\prime \prime} & D^{\prime}+p_{1} D^{\prime \prime} \\
D^{\prime}+p_{2} D^{\prime \prime} & 2 D^{\prime}+p_{2} D^{\prime \prime}
\end{array}\right)=\left(D^{\prime}\right)^{2}\left(3-2 \omega\left(D\left(p_{i}\right)\right)\right),
$$

where the right-hand side is positive by assumption. Applying Cramer's rule yields

$$
\frac{d p_{i}}{d f_{i}}=\frac{d p_{j}}{d f_{i}}=-\frac{\left(1-\omega\left(D\left(p_{i}\right)\right)\right)}{\left(3-2 \omega\left(D\left(p_{i}\right)\right)\right)} \cdot \frac{\partial \Gamma}{\partial f_{i}} .
$$

\footnotetext{
${ }^{19}$ Aguirre et al. (2010) use a similar convexity measure for demand functions.
} 
The right-hand side is strictly positive when $\omega\left(D\left(p_{i}\right)\right)<1$ in equilibrium $\left(\omega\left(D\left(p_{i}\right)\right)=\right.$ $\left.-p_{i} D^{\prime \prime} / D^{\prime}\right)$. Furthermore, an increase in own frequency leads to an increase in the sum of fares,

$$
\frac{d\left(p_{i}+p_{j}\right)}{d f_{i}}=-\frac{\left(2-2 \omega\left(D\left(p_{i}\right)\right)\right)}{\left(3-2 \omega\left(D\left(p_{i}\right)\right)\right)} \cdot \frac{\partial \Gamma}{\partial f_{i}}<-\frac{\partial \Gamma}{\partial f_{i}},
$$

but this increase is less than the reduction in schedule delays when $\omega\left(D\left(p_{i}\right)\right)<1$ in equilibrium, which is satisfied by standard assumptions on the curvature of the demand function. ${ }^{20}$ Altogether, these results establish the lemma.

\section{A.2 Proof of Lemma 3}

Using the first-order condition for optimal vehicle sizes (3) and with symmetry in frequency supply, the first-order condition $\partial E \pi_{i} / \partial k_{i}=0$ can be written as

$$
\frac{1}{4}\left(B^{\prime \prime}\left(k_{i} ; \sigma\right) k_{i}+B^{\prime}\left(k_{i} ; \sigma\right)-\Gamma\left(f_{1}, f_{2}\right)\right)-g^{\prime}\left(k_{i}\right)=0 .
$$

Totally differentiating this first-order condition with respect to capacity $k_{i}$ and schedule delay cost $\Gamma$ and applying the implicit function theorem yields

$$
\frac{d k_{i}}{d \Gamma}=\frac{1}{\left(2-\omega\left(B^{\prime}\left(k_{i}\right)\right)\right) B^{\prime \prime}-4 g^{\prime \prime}} .
$$

The right-hand hand side is negative in sign for $\omega\left(B^{\prime}\left(k_{i}\right)\right)<2$, which is a standard assumption on the convexity of the inverse demand function and ensures the existence of a solution. Finally, a joint increase in frequency supplies reduces schedule delay costs, i.e., $d k_{i} / d f_{1}+d k_{i} / d f_{2}=d k_{i} / d \Gamma \cdot 2 \partial \Gamma / \partial f_{i}>0$ for $f_{1}=f_{2}$, which completes the proof.

\section{A.3 Proof of Proposition 3}

To show that carrier collaboration increases frequency supply, it is useful to consider the unifying objective $E \pi_{1}+\phi E \pi_{2}$, where $\phi$ is called the collaboration parameter. For $\phi=0$, the objective is maximization of carrier 1's expected profit (this represents the independent carrier scenario), while the objective becomes maximization of joint expected profit for $\phi=1$ (this represents the alliance carrier scenario). Expected profits in (2) imply that own expected profit is independent of the other carrier's vehicle sizes, which leads to $\partial \pi_{i} / \partial s_{j}=0$ for $i=1,2$. Vehicle sizes and frequency supplies are therefore implicitly

\footnotetext{
${ }^{20}$ Czerny, Verhoef and Zhang $(2015)$ show that $\omega\left(D\left(p_{i}\right)\right)<1$ can be ensured for sufficiently concave marginal benefits, i.e., $\omega\left(B^{\prime}(q)\right)<-1 / B^{\prime \prime}(q)$.
} 
determined by the first-order conditions

$$
\begin{aligned}
& x_{1}=\frac{\partial E \pi_{1}}{\partial s_{1}}=0, \\
& x_{2}=\frac{\partial E \pi_{1}}{\partial f_{1}}+\phi \frac{\partial E \pi_{2}}{\partial f_{1}}=0 .
\end{aligned}
$$

Totally differentiating these first-order conditions with respect to the collaboration parameter, $\phi$, and using symmetry as well as $\partial E \pi_{i} / \partial s_{j}=0$ for $i=1,2$, yields the system of equations

$$
\left(\begin{array}{ll}
\frac{\partial x_{1}}{\partial s_{1}} & \frac{\partial x_{1}}{\partial f_{1}}+\frac{\partial x_{1}}{\partial f_{2}} \\
\frac{\partial x_{2}}{\partial s_{2}} & \frac{\partial x_{2}}{\partial f_{1}}+\frac{\partial x_{2}}{\partial f_{2}}
\end{array}\right)\left(\begin{array}{c}
d s_{1} \\
d f_{1}
\end{array}\right)=d \phi\left(\begin{array}{c}
-\frac{\partial x_{1}}{\partial \phi} \\
-\frac{\partial x_{2}}{\partial \phi}
\end{array}\right)
$$

with $\partial x_{1} / \partial \phi=0$. Before applying Cramer's rule, it is useful to denote

$$
\Phi=\operatorname{det}\left(\begin{array}{ll}
\frac{\partial x_{1}}{\partial s_{1}} & \frac{\partial x_{1}}{\partial f_{1}}+\frac{\partial x_{1}}{\partial f_{2}} \\
\frac{\partial x_{2}}{\partial s_{2}} & \frac{\partial x_{2}}{\partial f_{1}}+\frac{\partial x_{2}}{\partial f_{2}}
\end{array}\right)
$$

where the right-hand side is positive, i.e., $\Phi>0$, for sufficiently convex functional forms for capacity and schedule delay cost functions. To see this, note that $g^{\prime \prime}$ and $\partial^{2} \Gamma / \partial f_{i}^{2}$ enter $\partial x_{1} / \partial s_{1}$ and $\partial x_{2} / \partial f_{1}$, respectively, in such a way that $\Phi>0$ can be ensured when $\omega\left(g\left(k_{i}\right)\right)$ and $\omega\left(\Gamma\left(f_{1}, f_{2}\right)\right)\left(=f_{i} \cdot\left(\partial^{2} \Gamma / \partial f_{i}^{2}\right) /\left(\partial \Gamma / \partial f_{i}\right)\right)$ are sufficiently high in equilibrium.

Applying Cramer's rule further yields:

$$
\begin{aligned}
\frac{d f_{1}}{d \phi} & =-\frac{1}{\Phi} \frac{\partial^{2} E \pi_{1}}{\partial s_{1}^{2}} \frac{\partial E \pi_{2}}{\partial f_{1}} \\
\frac{d s_{1}}{d \phi} & =\frac{1}{\Phi}\left(\frac{\partial x_{1}}{\partial f_{1}}+\frac{\partial x_{1}}{\partial f_{2}}\right) \frac{\partial E \pi_{2}}{\partial f_{1}} .
\end{aligned}
$$

The right-hand side of (17) is strictly positive by the second-order conditions for best responses, which imply $\partial^{2} E \pi_{1} / \partial s_{1}^{2}<0$, and because an increase in the other carrier's frequency supply increases own demand and profit, i.e., $\partial E \pi_{2} / \partial f_{1}>0$. In contrast, the sign of the right-hand side in (18) is difficult to predict. This means that the effect of carrier collaboration on overall carrier capacity, $k_{i}$, can be positive or negative in principle. However, since carrier collaboration unambiguously increases frequency, carrier collaboration also increases optimal overall capacities $k_{i}$ by Lemma 3 (which shows that an increase in frequencies increases the optimal overall capacities $k_{i}$ ). 